\title{
Directional P-wave Remote Acoustic Imaging in an Acoustically Slow Formation
}

\author{
Zhoutuo Wei ${ }^{1}$, Hua Wang ${ }^{2, *}$, Xiaoming Tang ${ }^{3}$ and Chunxi Zhuan ${ }^{3}$ \\ ${ }^{1}$ School of Geosciences, China University of Petroleum, Qingdao, Shandong Province, 266580, China; ${ }^{2}$ State Key \\ Laboratory of Petroleum Resources and Prospecting, China University of Petroleum, Beijing, 102249, China; ${ }^{3}$ School \\ of Geosciences, China University of Petroleum, Qingdao, Shandong Province, 266580, China
}

\begin{abstract}
Directional P-wave remote acoustic imaging in an acoustically slow formation is discussed to improve dipole remote acoustic applications. In this paper, we start from the fundamental radiation, reflection and reception theory of a borehole dipole source. We then simulate the elastic wavefield radiation, reflection and reception generated by a borehole dipole source in an acoustically slow formation, and analyze their similarities and differences of the far-field radiation directionality of a borehole dipole-generated P-wave and monopole-generated P-wave. We demonstrate its sensitivity and feasibility in conjunction with a numerical simulation of $\mathrm{P}$-wave remote acoustic imaging. The analytical results show that the dipole-generated P-wave has obvious reflection sensitivity and it can be utilized for reflection imaging and determination of the reflector azimuth. Based on the theoretical analysis above, a field example is used to demonstrate these characteristics and the application effectiveness of dipole-generated P-wave imaging and monopole-generated P-wave imaging. The results substantiate that dipole-generated $\mathrm{P}$-wave has highly reflected amplitude and obvious azimuth sensitivity in an acoustically slow formation, providing an important supplement for dipole-generated S-wave remote acoustic imaging.
\end{abstract}

Keywords: Dipole source, Numerical simulation, P-wave imaging, Wavefield distribution.

\section{INTRODUCTION}

Along with the development of oil-gas exploration and production and borehole acoustic measurements, it is usually necessary to know near-borehole geological structures. Remote acoustic imaging technology is ideal for this need. It can broaden the scope of borehole acoustic measurement from about one meter to several tens of meters, giving rise to several important applications. For example, it can provide subsurface geological structural features at a resolution impossible to achieve with surface seismic, playing an increasingly important role in imaging structural boundary, detecting near-borehole fractures and fault crossing the borehole, mapping salt dome internal structures, tracing reservoir boundaries in a horizontal well and even serving as a geosteering tool [1-8].

There are heretofore two methods about the remote acoustic imaging, one is the monopole P-wave method, and the other is dipole S-wave method. The former uses P-wave acoustic energy that radiates away from the borehole and reflects back to the borehole from near-borehole reflector to determine its spatial position and azimuth. It is mostly performed exclusively with a monopole acoustic measurements represented by Schlumberger's BARS (Borehole Acoustic Reflection Survey) technology. Because of the omnidirectional radiation of monopole source, the reflected wave may come from anywhere from the $360^{\circ}$ azimuth around the

*Address corresponding to this author is at Earth Resources Laboratory at Massachuset Institute of Technology, Cambridge, MA, 02139, USA;

Tel: 1-617-253-0950; Fax: 1-617-253-6385;

E-mail: wanghuaupc@126.com borehole. It obtains only a 2D image of near-borehole reflectors, and cannot provide the azimuthal information of a nearborehole structure. In order to improve the deficiency of azimuthal uncertainty, Schlumberger has designed a SonicScanner tool with a directional sonic imaging function and has utilized the receiver station configuration in the design of the tool. Even so, its ability to detect near-borehole reflectors is limited because of its limited penetration depth, just from several meters to tens of meters, due to the use of highfrequency P-wave $(8-10 \mathrm{kHz})$. Compared to the monopole source, the lower frequency nature $(2-5 \mathrm{kHz})$ of a borehole dipole source operation allows for a deeper penetration depth into the formation (several tens of meters range), and the strike of reflectors can be determined by the directionality attribute of the dipole measurement. Tang [9] used acoustic waves from a directional acoustic tool to image a formation structure and explored the directional aspect of P-wave generated by a dipole acoustic tool. Afterwards, some work has been done on the use of dipole acoustic wave for remote acoustic imaging. However, these researches primarily focused on the S-wave processing aspects [8-10]. For dipole Pwave remote imaging, it remains to have a detailed analysis on the radiation, reflection and reception of the elastic waves generated by a dipole source. Especially for those slow formations, remote acoustic imaging of dipole S-wave usually needs a lengthy record in the process of the acoustic logging. However, using extended recording time will render reflected S-wave over attenuated. Dipole P-wave imaging can fully meet requirements under the conventional acoustic logging, and serve as an important supplement to the dipole remote acoustic imaging method. 
In order to understand and utilize fully P-wave remote acoustic imaging, we first proceed from fundamental theory of the radiation, reflection and reception of the wavefield of a borehole dipole source. We then simulate the elastic wavefield radiation, reflection and reception generated by a borehole dipole source (wavefield in and outside the borehole), and compare similarities and differences of dipole source far-field radiation directionality of a dipole P-wave and monopole P-wave. In particular, we confirm the dipole Pwave sensitivity and feasibility in conjunction with a numerical modeling analysis. Essentially, by comparing imaging results of a dipole P-wave and monopole $\mathrm{P}$-wave, we find the concrete embodiment of these characteristics and application effect of dipole P-wave remote imaging. Finally, we draw some conclusions.

\section{THEORY AND METHOD}

According to the method and results of [10] for a lowfrequency dipole source in a fluid-filled borehole, when a dipole acoustic source on the tool is fired, it radiates three types of elastic waves into the formation surrounding the borehole: P-wave, the polarized SV-wave in the plane containing the borehole, and the polarized SH-wave normal to the plane containing the borehole, respectively. Fig. (1) shows a dipole source in a fluid-filled borehole generates wave propagation along borehole and radiates elastic waves into formation. The radiated waves propagate outwards from the borehole and reflect back to the borehole from the nearborehole reflectors, received by geophones equipped within the borehole. Combining the wave radiation, reflection [11], attenuation, and borehole reception [12], we can write the P-, $\mathrm{SV}-$, and SH-wave displacement components in borehole as

$$
\left\{\begin{array}{l}
u_{P}=\frac{S(\omega)}{4 \pi \rho \alpha^{2}} \cdot \frac{e^{i \omega D / \alpha}}{\mathrm{D}} \cdot e^{-\omega D / 2 \alpha Q_{\alpha}} \cdot R F_{p} \cdot \cos \theta \cos \theta_{1} \sin \varphi=P \sin \varphi \\
u_{S H}=\frac{S(\omega)}{4 \pi \rho \beta^{2}} \cdot \frac{e^{i \omega D / \beta}}{\mathrm{D}} \cdot e^{-\omega D / 2 \beta Q_{\beta}} \cdot R F_{s h} \cdot \cos \varphi=S H \cos \varphi \\
u_{S V}=\frac{S(\omega)}{4 \pi \rho \beta^{2}} \cdot \frac{e^{i \omega D / \beta}}{\mathrm{D}} \cdot e^{-\omega D / 2 \beta Q_{\beta}} \cdot R F_{s v} \cdot \cos \theta \cos \theta_{1} \sin \varphi=S V \sin \varphi
\end{array}\right.
$$

where $\alpha, \beta$ and $\rho$ are formation P-wave velocity, Swave velocity and the solid density, respectively; $S(\omega)$ is the source spectrum; $R F_{p}, R F_{s h}$ and $R F_{s v}$ are the $\mathrm{P}-, \mathrm{SV}-$, and $\mathrm{SH}$-wave reflection coefficient at the reflector, respectively; $D$ is the total travel distance from the source to the reflector, and from the reflector to the receiver; $\varphi$ is the angle of the wave incidence plane from the strike angle of the reflector; $\theta$ and $\theta_{1}$ are the radiation wave take-off angle and reflection wave incident angle to the borehole, respectively; $Q_{\alpha}$ and $Q_{\beta}$ are the formation $\mathrm{P}$ - and $\mathrm{S}$-wave quality factors, respectively; The symbols P-, SV-, and SH- represent the P-, SV-, and SH-wave reflection displacement when the source orientation is normal to (for P- and SV-wave) and parallel with (for SH-wave) the reflector strike, respectively.

Tang and Patterson [10] derived the far-field S-wave radiation of a low frequency dipole source in a fluid-filled borehole. It is different from that of far-field P-wave radiation [13] as follows:
$\left\{\begin{array}{l}u_{R}=\Gamma \cdot \frac{S(\omega)}{4 \pi \alpha^{2} D} \cdot\left[1-\left(2 \frac{\beta^{2}}{\alpha^{2}}-\frac{\beta^{2} / \alpha^{2}}{\rho_{f} / \rho+\beta^{2} / \alpha_{f}^{2}}\right) \cos ^{2} \theta\right] e^{i \omega D / \alpha} \\ u_{\theta}=2 \Gamma \cdot \frac{S(\omega)}{4 \pi \beta^{2} D} \cdot e^{i \omega D / \beta} \sin \theta \cos \theta\end{array}\right.$

Where $\Gamma=\frac{1}{\rho_{f} / \rho+\beta^{2} / \alpha_{f}^{2}} \cdot \frac{\rho_{f}}{\rho} ; \alpha_{f}$ and $\rho_{f}$

are fluid velocity and density, respectively.

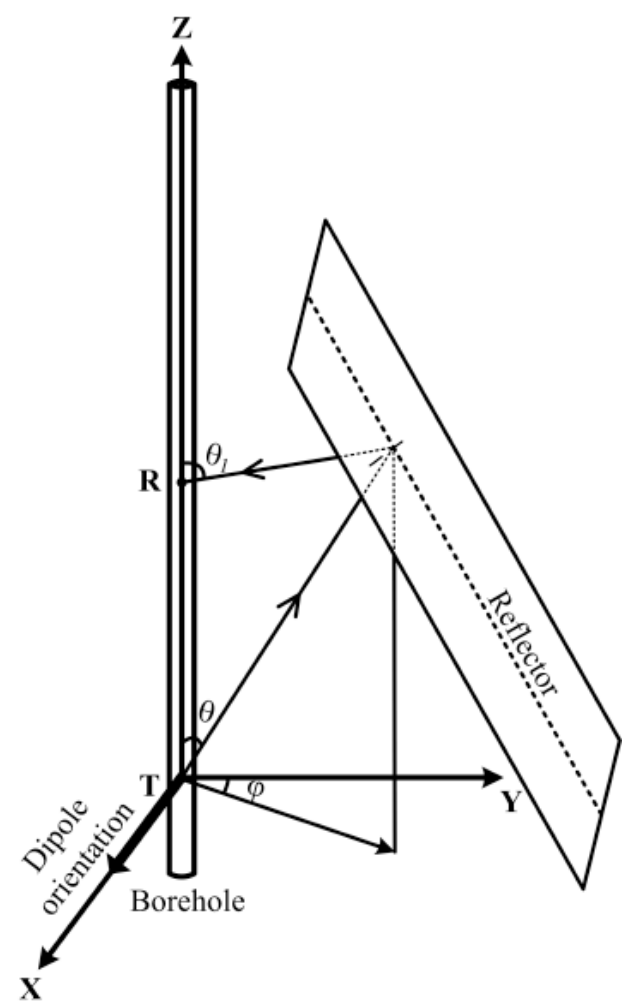

Fig. (1). A dipole source in a fluid-filled borehole generates wave propagation along borehole and radiates elastic waves into formation. A cylindrical coordinate system is used to analyze the farfield radiation of a dipole source in a fluid-filled borehole.

Comparing equation 1 and equation 2, it can be seen that both of radiation field with the distance of the geometric spreading factor are 1/D, and their radiation fields spread outside in a spherical wave form. One main difference is that dipole radiation field from the acoustic source contains $\mathrm{SH}-$ wave without a $\theta$ dependence and therefore with a wide radiation pattern, while the $\mathrm{P}$-wave generated by a dipole source has azimuth directivity. Far-field radiation of a dipole source does not have dependence on the borehole fluid properties. However, far-field radiation of a monopole source is related to fluid-filled borehole. The reason is that the acoustic pressure generated by a monopole source depends on fluid volume variation. In comparison, the overall fluid volume variation of dipole source in borehole is zero, and thus the wavefield has little fluid dependence.

In the field acoustic measurement, two orthogonal dipole source-receiver systems are used, where one system is oriented in the $x$-axis direction and the other is in the $y$-axis 
direction. Project the S-wave onto the $x$ - and $y$-axis directions of the receivers to give the $x x_{p}$ and $x y_{p}$ components data, respectively. This paper discusses only two P-wave components in the $x$ - and $y$-axis directions

$$
\left\{\begin{array}{l}
x x_{P}=P \sin ^{2} \varphi \\
x y_{P}=P \sin \varphi \cos \varphi
\end{array}\right.
$$

Performing the same analysis for the $y$-dipole source of the same intensity $P$, we get the $y x$ - and $y y$-component data

$$
\left\{\begin{array}{l}
y y_{P}=P \cos ^{2} \varphi \\
y x_{P}=P \sin \varphi \cos \varphi
\end{array}\right.
$$

By comparising equation 3 and equation 4 , we need only the $x x_{P}$ and $y y_{P}$ components to determine the maximum reflected P-wave amplitude (i.e., the dipole source is oriented along the reflector plane)

$$
P=x x_{P}+y y_{P}
$$

The angle $\varphi$ can be determined by comparing the relative amplitude between $x x_{P}$ and $y y_{P}$ components.

\section{NUMERICAL SIMULATION AND RESULTS ANAL- YSIS}

\section{Circumferential Wavefield Distribution Characteristics of Different Source Types Outside a Fluid-filled Borehole}

In order to understand the far-field radiation directivity characteristics by different source excitation, Fig. (2a) shows sketch of circumferential receiver arrays. In a Cartesian coordinate system $x, y, z$, the model dimension is $10 \times 10 \times 10 \mathrm{~m}$, with a $0.1 \mathrm{~m}$ radius fluid-filled borehole. The center of source is located at $x=y=z=5 \mathrm{~m}$. Two receiver circles, each of 122 receivers spaced at every $3^{\circ}$ on the circumferential receiver array, are placed at $4.0 \mathrm{~m}$ distance from borehole in the $x o z$ and yoz orthogonal plane. Fig. (2b) shows spatial configuration of the circumferential receivers. The simulation uses a Kelly source [14] of $3 \mathrm{kHz}$ center frequency for the dipole source and of $8 \mathrm{kHz}$ center frequency for the monopole source at the borehole center, respectively.

Fig. (3) shows wavefield distribution of yoz and $x o z$ plane in fluid-filled borehole, and borehole axis along $z$-axis. Dipole source points to $x$-axis, as shown in Fig. (3a). In the $y-z$ vertical plane, we observe a pure $\mathrm{SH}$-wave and a high amplitude flexural wave due to borehole excitation along the $z$-axis. The SH-wave amplitude is the highest along the $y$ axis and diminishes away from it. Fig. (3b) shows P-wave and $\mathrm{SV}$-wave radiation pattern characteristics in the vertical $x-z$ plane. By the same token, we observe high amplitude flexural wave of borehole excitation along the $z$-axis. Obviously, the radiated $\mathrm{P}$-wave and $\mathrm{SV}$-wave outside borehole have angle dependence from the $z$-axis, and both amplitudes are nearly null at the $x$-axis and $z$-axis, respectively. Fig. (3d) shows calculated $\mathrm{SH}$-wave, $\mathrm{SV}$-wave and $\mathrm{P}$-wave radiation patterns from the wavefield data (Fig. 3a and 3b). As can be seen from Fig. (3d), SH-wave is no longer a circular pattern. Compared with a lower frequency, the wavelength is only twice compared to the borehole size in a slow formation, and the borehole scattered energy is not negligible compared to the incident wave when the logging frequency is about several thousand Hertz. The SV-wave and P-wave radiation patterns ( $x o z$ plane) both approach zero amplitude as the incident angle approaches $90^{\circ}$ and $0^{\circ}$, respectively. However, SV-wave at normal incidence to the borehole cannot be detected and P-wave at normal incidence is the same with $\mathrm{SH}$-wave. When the incident angle approaches $0^{\circ}$, the nearborehole reflector is perpendicular to the borehole, and wave reflection occurs only from one point. Actually, both

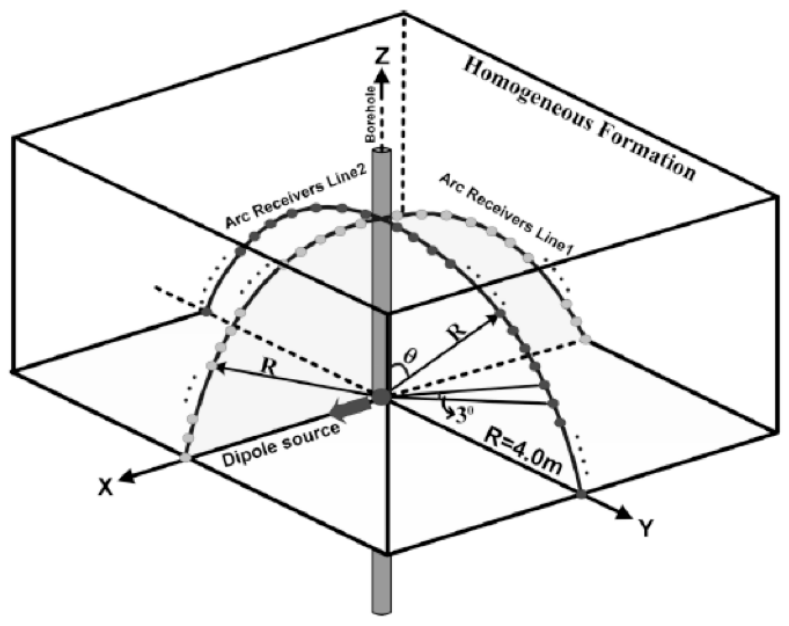

(a)

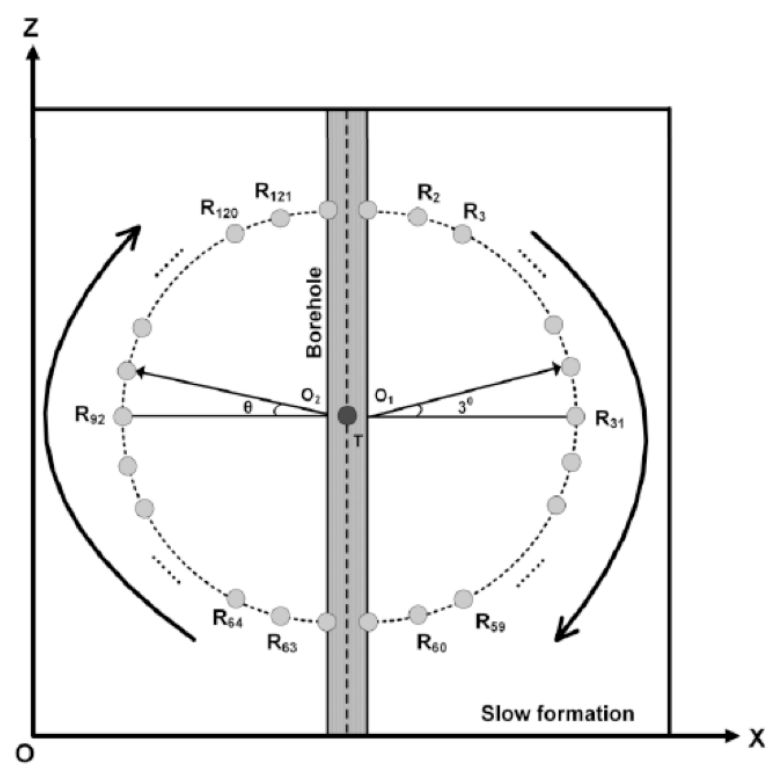

(b)

Fig. (2). Calculated wavefield distribution model with a fluid-filled borehole in two orthogonal planes. (a) Schematic diagram of far-field radiation of a dipole source in a fluid-filled borehole, (b) Receivers layout in the yoz and xoz plane. 


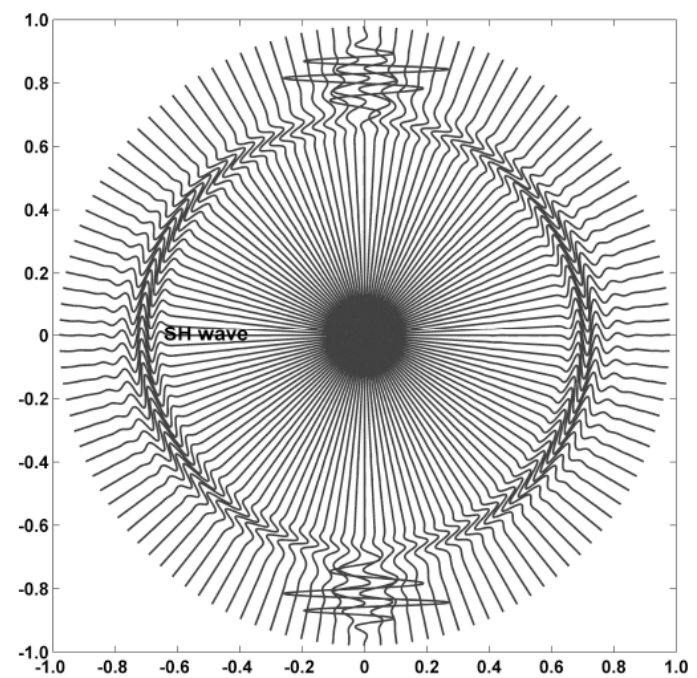

(a)

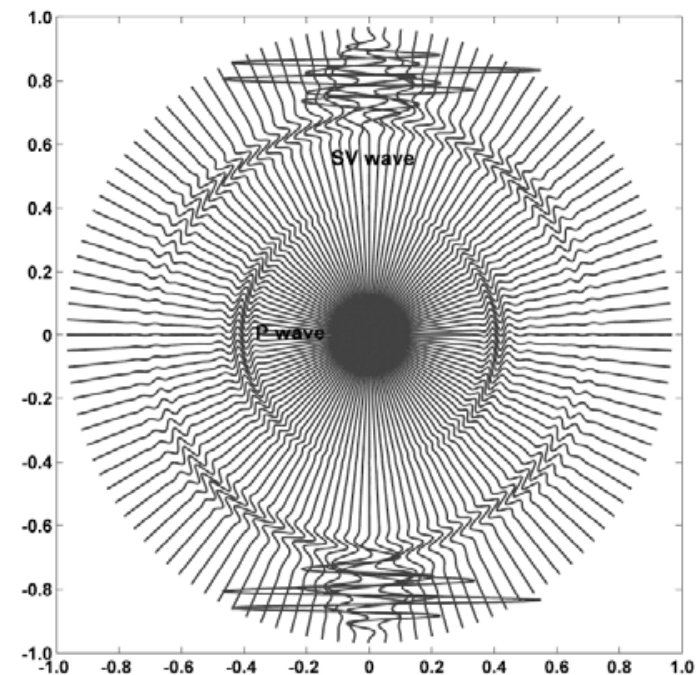

(b)

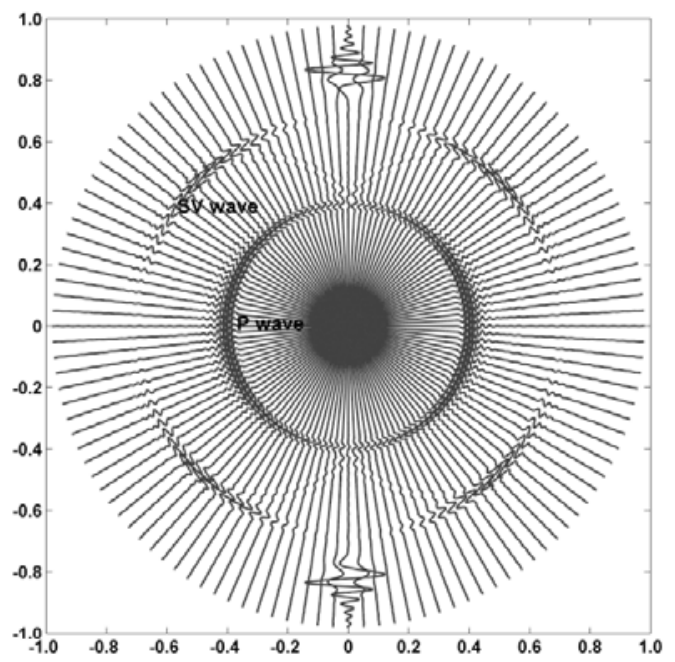

(c)

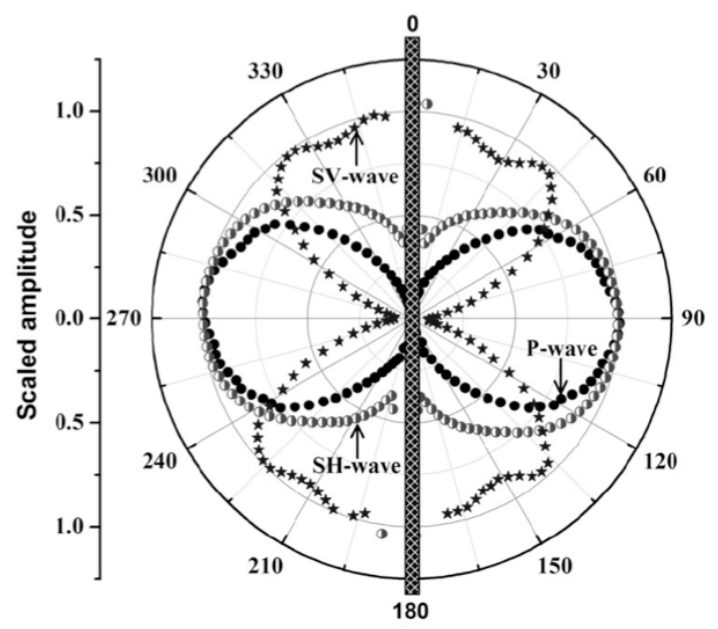

(d)

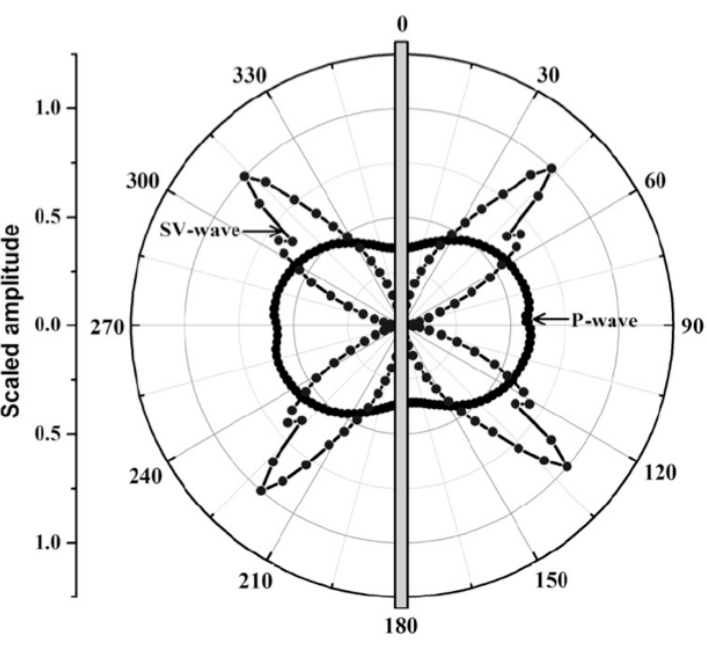

(e)

Fig. (3). Calculated wavefield distribution with a fluid-filled borehole in two orthogonal planes. In this figure, the vertical and horizontal coordinates are the displacement relative amplitudes and are dimensionless. (a) dipole SH-wave in the yoz plane, (b) dipole P-wave and SVwave in the xoz plane, (c) monopole P-wave and SV-wave in the xoz (yoz) plane, (d) radiation directivity of dipole P-wave SH-wave and SVwave, (e) far-filed radiation directivity of monopole P-wave and SV-wave. 
SH-wave and P-wave can be imaged and both angular coverages are in the $45^{\circ}-135^{\circ}$. In general, the radiated $\mathrm{SH}$-wave, $\mathrm{P}$-wave and $\mathrm{SV}$-wave outside the borehole have angle dependence from the $z$-axis. Fig. (3c) shows wavefield distribution generated by a monopole source in a fluid-filled borehole, where the P-wave and SV-wave amplitude characteristics are identical in the $x o z$ and yoz vertical planes. Fig. (3e) shows the SV-wave amplitude is null at the $x$-axis and $z$-axis. There are four nodal planes. P-wave radiation coverage from the dipole source is also wider than that of a monopole SVwave.

Through the comparisons above we find a monopole source has a omni-directional azimuthal radiation for $\mathrm{P}$ wave, and all receivers located on the tool can record wave energy from all azimuthal directions. Therefore, it obtains only a 2D image of near-borehole reflectors in 3D space. As shown in Fig. (3d), the far-field radiation pattern of a dipole source possesses obvious azimuthal sensitivities. The operating lower frequency and transmitting power of the borehole dipole source allow for deeper penetration depth. In addition, $\mathrm{S}$-wave remote acoustic imaging usually requires a lengthy record in the process of the acoustic logging in a slow formation. However, the dipole-generated P-wave can fully meet the requirements under conventional logging, and it can be used for remote acoustic imaging.

\section{Simulation of Dipole Wave Reflection for a Near- borehole Reflector in an Acoustically Slow Formation}

We now simulate the reflection from a near-borehole reflector in a slow formation and analyze the reflection wave variation with the included angle of dipole source orientation and reflector strike. Fig. (4) shows a 3D simulation model with a near-borehole reflected interface, where the reflector incidence angle is $80^{\circ}$. The model dimension is $8 \times 8 \times 8 \mathrm{~m}$, with a $0.1 \mathrm{~m}$ radius fluid-filled borehole located at $x=y=1.0$ $\mathrm{m}$. The source is placed at $z=0.3 \mathrm{~m}$. To facilitate the discussion of the influence of the included angle on the reflected wavefield, we build a $5 \mathrm{~m}$ radius quadrant, with its center located at $x=y=1 \mathrm{~m}$ (project borehole axis onto the xoy plane), move the interface of incidence angle of $80^{\circ}$ near the borehole according to the arrow shown in Fig. (4). The five positions are $\varphi=90^{\circ}, \varphi=60^{\circ}, \varphi=45^{\circ}, \varphi=30^{\circ}$ and $\varphi=0^{\circ}$ (measured relative to $y$-axis), respectively. $x$-oriented and $y$ oriented dipole source are fired at each interface position, respectively. We can get the $x x$-, $x y-, y y$ - and $y x$-component. The simulation uses a dipole source with $3 \mathrm{kHz}$ center frequency, and the source type is the same as above. The borehole and the elastic property on both sides of the reflected interface are given in Table $\mathbf{1}$.

In order to understand the simulation results, we perform a wavefield snapshot analysis for the xoz and yoz planes at different moments. Tang and Patterson [10] analyze the Swave radiation of a borehole dipole source and point out when the source orientation is along the reflector strike direction $\left(\varphi=0^{\circ}\right)$, an $\mathrm{SH}$-wave reflection will occur. Fig. (5a) shows the snapshot at $\mathrm{T}=4.95 \mathrm{~ms}$ in the yoz section crossing fluid-filled borehole. The area inside the ellipse shows a marked reflected $\mathrm{SH}$-wave and it shows no interference from wave conversion. When the source points to the reflector plane $\left(\varphi=90^{\circ}\right)$, both P- and SV-wave reflections will be gen- erated. Fig. (5b and 5c) show the snapshot at $\mathrm{T}=2.85 \mathrm{~ms}$ and $\mathrm{T}=4.95 \mathrm{~ms}$ in the $x o z$ section crossing fluid-filled borehole, respectively. P-wave is the first arrival, shown by the ellipse the Fig. (5b). When the wavefield continues to propagate to $\mathrm{T}=4.95 \mathrm{~ms}$, the area inside the marked ellipse in Fig. (5c) shows a weak but discernable SV-wave reflection. At the moment, P-wave has already reflected back to the receiver array. Comparing these three cases, their relationship is $\mathrm{AMP}_{\mathrm{SH}}>\mathrm{AMP}_{\mathrm{PP}}>\mathrm{AMP}_{\mathrm{SV}}$ and $\mathrm{AMP}_{\mathrm{SP}+\mathrm{PS}} \approx 0$ (see equation 2 ). These characteristics are consistent with the previous analysis, demonstrating the benefit of using dipole-generated $\mathrm{P}$-wave for reflection imaging in the slow formation.

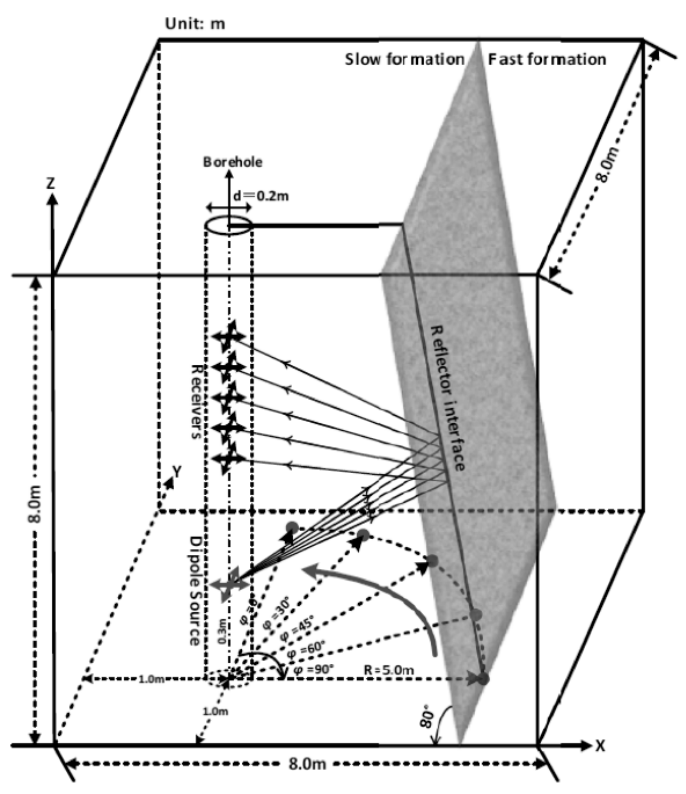

Fig. (4). 3D simulation model with a near-borehole reflector. Source and receiver configuration with respect to the reflector at various positions along a quarter circle.

Fig. (6) shows the simulated full waveforms in the $x x$ and $y y$-component in the fluid-filled borehole for the five included angles. The receiver oriented in the source direction is located on the borehole axis $2.0 \mathrm{~m}$ above the source. For comparison, the bottom waveform shows the simulated fullwave computed for a homogeneous formation without the reflector interface. For all five cases, as well as for the homogeneous formation case, the first arrival is the same Pwave propagating along the borehole, which may also be called Leaky-P wave [15]. A remarkable phenomenon is the large P-to-P reflected wave and its amplitude variation with from weakest $\left(\varphi=0^{\circ}\right)$ to strongest $\left(\varphi=90^{\circ}\right)$, as highlighted by the middle rectangular in Fig. (6a). However, the variation has opposite trend in Fig. (6b) compared to that of the reflected P-wave. It can be seen that reflected P-wave has obvious azimuthal sensitivities. The large amplitude event in Fig. (6) is the dipole flexural wave traveling along the borehole. In particular, to explain the striking reflected $\mathrm{P}$-wave in a slow formation, we set up a model which has a P- to Swave velocity ratio of 1.84 and Poisson's ratio of 0.2904 . We can see that the P-to-P reflected wave arrives after the flexural wave but its amplitude is still significant. For higher Poisson's ratio in acoustically slow formation (as shown in field application), reflected $\mathrm{P}$-wave amplitude will be more 
Table 1. Physical properties of media used in the numerical simulation. The borehole radius is $0.1 \mathrm{~m}$.

\begin{tabular}{|c|c|c|c|}
\hline Medium & $\begin{array}{c}\text { P-wave velocity } \\
(\mathbf{m} / \mathbf{s})\end{array}$ & $\begin{array}{c}\text { S-wave velocity } \\
(\mathbf{m} / \mathbf{s})\end{array}$ & $\begin{array}{c}\text { Density } \\
\left(\mathbf{k g} / \mathbf{m}^{\mathbf{3}}\right)\end{array}$ \\
\hline \hline Borehole fluid & 1500 & 1250 & 1000 \\
\hline Slow formation & 2300 & 2000 & 1800 \\
\hline Fast formation & 3800 & 2650 & 2150 \\
\hline Fast formation1 & 4500 & 2400 \\
\hline
\end{tabular}

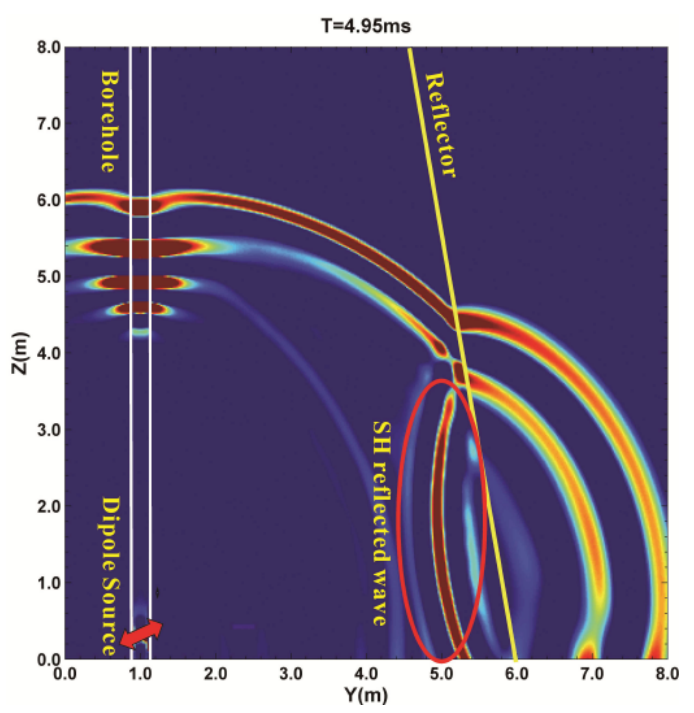

(a)

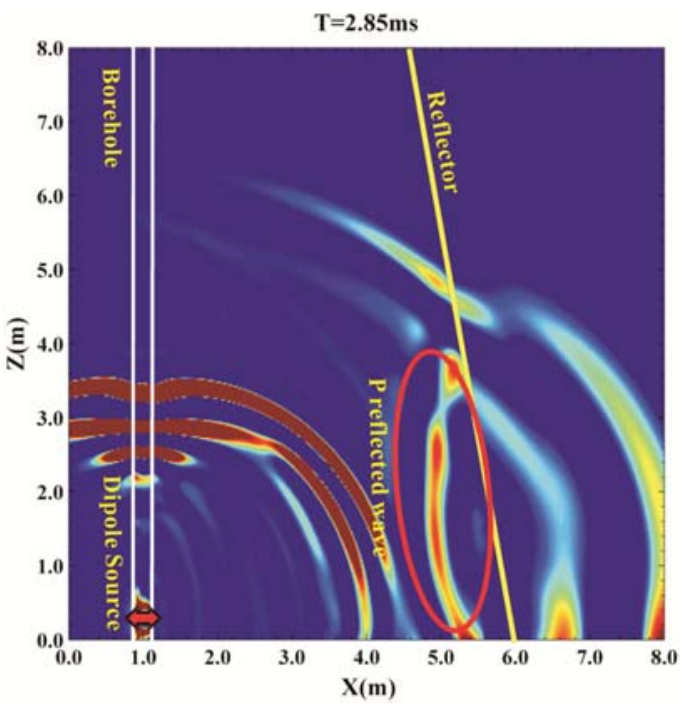

(b)

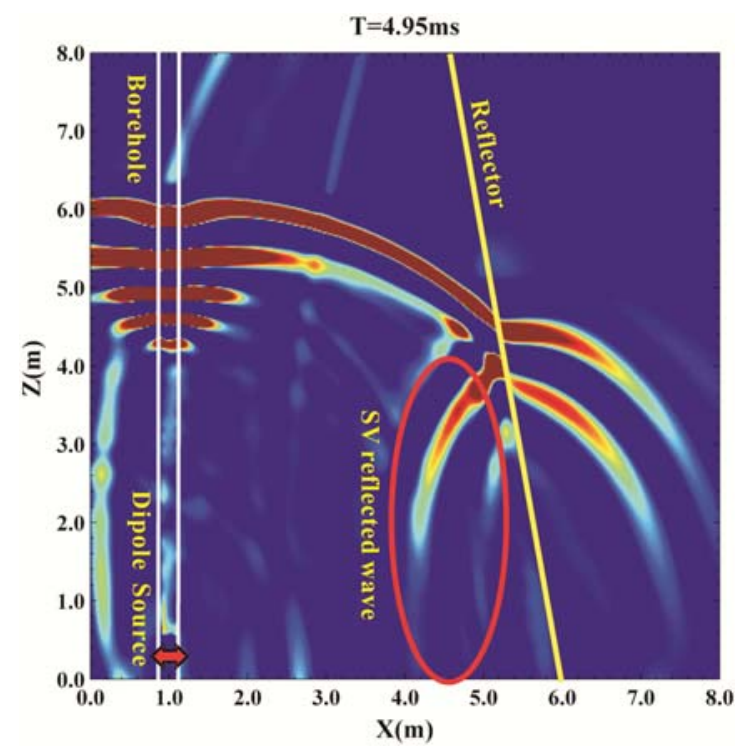

(c)

Fig. (5). Snapshots of dipole-generated wavefield in the $x o z$ and $y o z$ sections at different times. (a) $v_{x}$ component is shown at $T=4.95 \mathrm{~ms}$ and the reflector is $\varphi=0^{\circ}$ of Fig. (4), generating a pure SH-wave reflection; (b) $v_{x}$ component is shown at $T=2.85 \mathrm{~ms}$ and the reflector is $\varphi=90^{\circ}$ in Fig. (4), generating a P-wave reflection; (c) $v_{x}$ component is shown at $\mathrm{T}=4.95 \mathrm{~ms}$ and the reflector is $\varphi=90^{\circ}$ of Fig. (4), generating a SV-wave reflection.

significant, and their relationship becomes $\mathrm{AMP}_{\mathrm{PP}}>\mathrm{AMP}_{\mathrm{SH}}$ $>\mathrm{AMP}_{\mathrm{SV}}$ [16]. For Fig. (6a and $\left.\mathbf{6 b}\right)$, the later arrival is the Sto-S reflected wave from the near-borehole reflector, as highlighted by the right rectangle in the figures. The S-to-S re- flected wave also shows significant amplitude variation with the included angle. The variation trend is opposite to that of the reflected P-wave. 


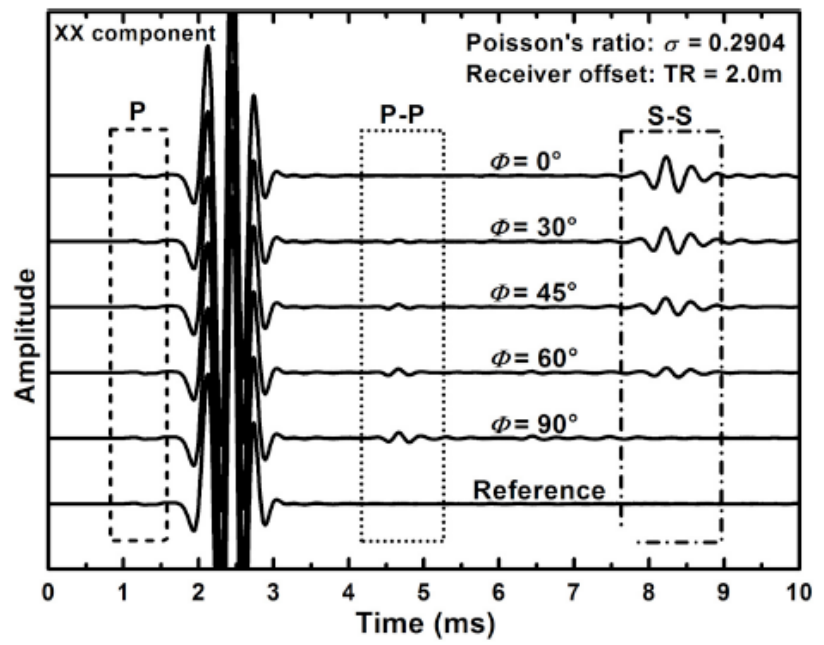

(a)

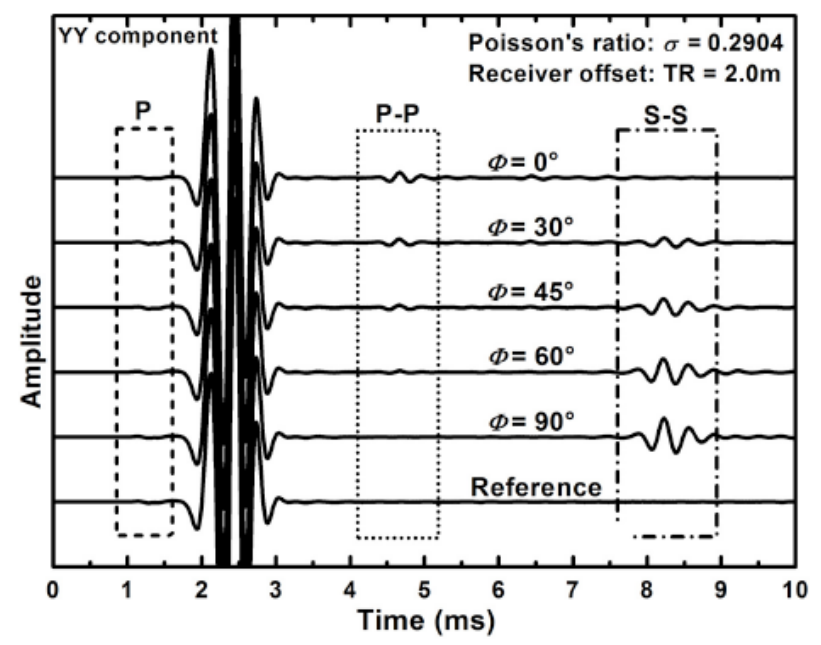

(b)

Fig. (6). Simulated full waveform from the dipole source in a fluidfilled borehole for different reflector positions (a) $x x$-component, (b) yy-component.

The above analysis shows that P-wave generated by a dipole acoustic tool in slow formation possesses high amplitude, obvious sensitivity, and wide coverage characteristics. Consequently, P-wave generated by a dipole source can also be used for remote reflection imaging, providing the theoretical basis for dipole $\mathrm{P}$-wave remote acoustic imaging in an acoustically slow formation.

Tang et al. [10] developed processing techniques to determine reflector azimuth and to image the reflector. For the dipole P-wave, we need only to compare the relative amplitude between $x x_{P}$ and $y y_{P}$ to determine the angle $\varphi$. Therefore, we calculate these reflected P-wave amplitudes from $x x$-component of Fig. (6a) and $y y$-component of Fig. (6b) using $A_{\text {reflection }}=\|S(t)\|_{2} / \sqrt{N}$, respectively, where $S(t)$ is the amplitude of the reflected wave, $\|S(t)\|_{2}$ is 2-norm, $N$ is the signal sample and $A_{\text {reflection }}$ is the amplitude of the reflected wave. For the convenience of comparison, we also give the theoretical results (as indicated by dotted line in Fig. 7a). The computation results show that both are fully consistent with the theoretical analysis. Reflected P-wave amplitude versus the angle $\varphi$ for $x x$-component exhibits a $\sin ^{2} \varphi$ relationship, and amplitude for $y y$-component exhibits a $\cos ^{2} \varphi$ relationship (see Fig. 7a). The numerical results are very close to the theoretical results predicted by equations 3 and 4 . The figure shows also the maximum reflected $\mathrm{P}$-wave amplitude when the source orientation is normal with the reflector strike. It can be seen that we need only two components to determine the maximum reflected $\mathrm{P}$-wave amplitude in any case (equation 5). By solving the amplitude ratio of the $x x_{P}$ and $y y_{P}$ components, we calculate reflector azimuth $\varphi$. As can be seen from the figure, the computation results agree reasonably well with the forward model azimuth (see Fig. 7a and Fig. 7b).

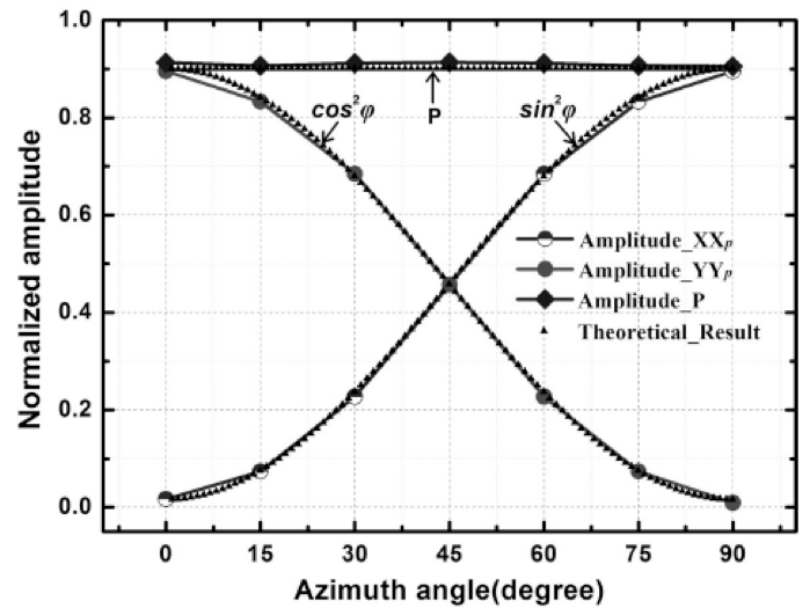

(a)

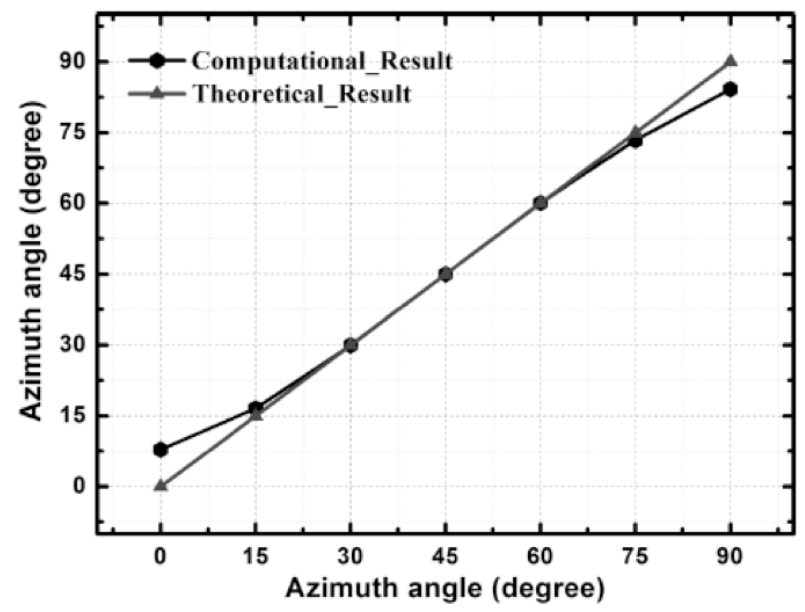

(b)

Fig. (7). Determining reflector azimuth. (a) reflected P-wave amplitude of $x x$-component of Fig. (6a) and yy-component of Fig. (6b) for different reflector positions, (b) reflector azimuth comparison between the computational result and the theoretical result.

\section{FIELD DATA APPLICATION}

Finally, we use an example of conventional 4-C crossdipole data and monopole data to demonstrate the dipole radiation and the reflected $\mathrm{P}$-wave characteristics described 


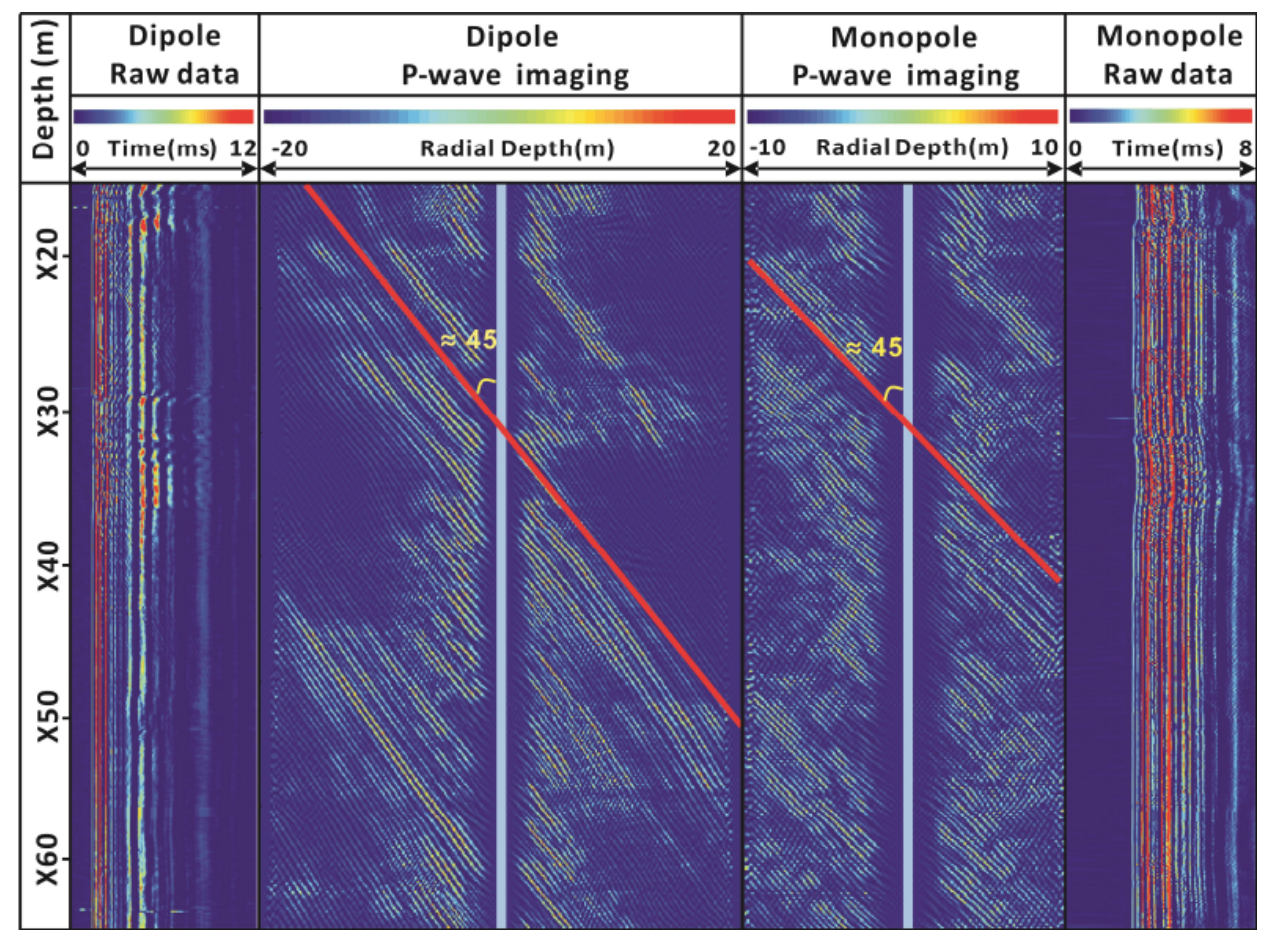

Fig. (8). Comparison of imaging results using the dipole-generated P-wave and the conventional monopole-generated P-wave.

above. In the meantime, we compare and analyze the acoustic imaging results of dipole $\mathrm{P}$-wave and monopole $\mathrm{P}$-wave.

Fig. (8) shows the comparison of the reflected P-wave image from the dipole data (yy-component) and that from the conventional monopole P-wave data. The acoustic-logging data sets, which are from routine cross-dipole logging and conventional monopole acoustic logging, respectively, are acquired in a $45^{\circ}$ deviation borehole penetrating an acoustically slow formation. In the logging process, the $x$-direction dipole is placed in the horizontal plane and the $y$-direction dipole is placed in the vertical plane. $\mathrm{P}$-wave and $\mathrm{S}$-wave velocity of the slow formation are $2200 \mathrm{~m} / \mathrm{s}$ and $800 \mathrm{~m} / \mathrm{s}$, respectively. Only dipole and monopole raw data from receiver one of an eight-receiver array are displayed in track 1 and 4 of Fig. (8), respectively. Both receiver raw data show some weak traces of reflection events.

In this slow formation, S-wave remote acoustic imaging needs a record of extended period of time in the acoustic logging process, while dipole-generated $\mathrm{P}$-wave imaging can fully meet requirements under the conventional acoustic logging. Based on the theoretical and numerical simulation results above, we can use reflected P-wave for remote reflection imaging. For the dipole data, we use the $y y$-component data for the reflected P-wave imaging, and the processing frequency range is $2-5 \mathrm{kHz}$. Imaging result is shown in track 2 of Fig. (8). The imaging result reveals a series of clear and legible formation boundaries crossing the borehole at about $45^{\circ}$ (Given in the form of variable density log map). The penetration depth reached more than $20 \mathrm{~m}$ into the formation.

Yet it is worth pointing out that there is no manifestation of reflected interface using imaging result of dipolegenerated $\mathrm{P}$-wave data from $x x$-component data (for the purpose of saving space, imaging results figure of $x x$-component is not shown). The results are obviously caused by the directionality effect of the dipole measurement. As equation 3 and Fig. (7a) show, when dipole source orientation ( $x$-direction) is parallel to a horizontal reflector, there is no reflected Pwave generated. And when the dipole source ( $y$-direction) is oriented along the reflector, it generates reflected P-wave. Similarly, we use monopole data for the P-wave imaging, as shown in track 3 of Fig. (8). It yields almost the same imaging result compared to the dipole case, indicating the accuracy and reliability of our imaging processing method. Compared to the dipole imaging result, these formation boundaries have a limited radial penetration depth about $10 \mathrm{~m}$ or one half that of the dipole image due to the higher frequency source signature. More importantly, the greatest disadvantage of the monopole $\mathrm{P}$-wave imaging is that it cannot determine the strike of near-borehole structures. In conclusion, the example substantiates that $\mathrm{P}$-waves generated by a dipole acoustic tool possess high amplitude and obvious sensitivity. It greatly enhances the radial penetration depth and determines readily the reflector strike.

\section{DISCUSSION}

\section{Reflected P-wave Amplitude Comparison}

As compared to a fast formation, the dipole generated Pwave energy is prominent and the imaging performs better in a slow formation. Fig. (9a) shows the relationship between the reflected $\mathrm{P}$-wave amplitude and the receiver offset for various source-reflector orientations, as well as for the maximum reflected P-wave amplitude in the fast formation. The amplitudes are normalized by the largest value of the reflected P-wave curve for the slow formation case. For brevity, only the largest reflected $\mathrm{P}$-wave amplitude in the fast formation is plotted. It can be seen both reflected P-wave amplitude with receiver offset increase gradually, reach to 


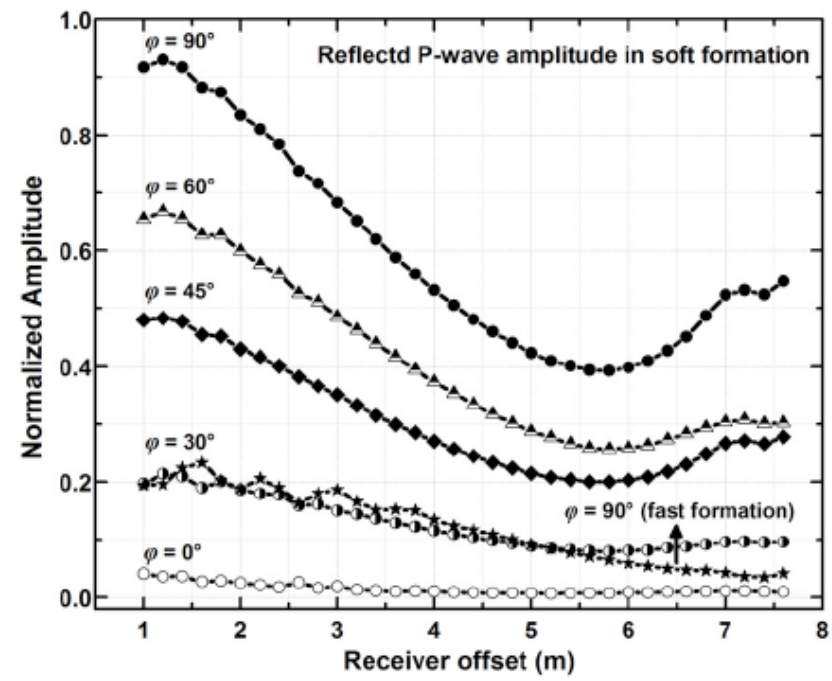

(a)

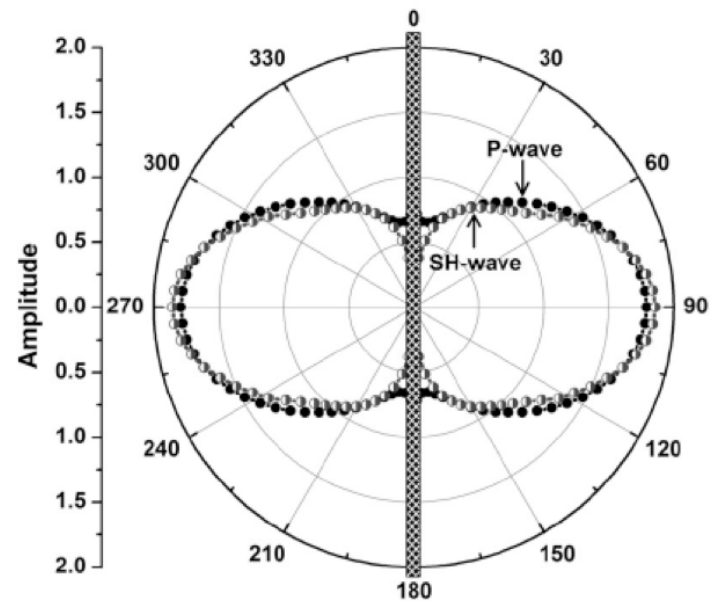

(b)

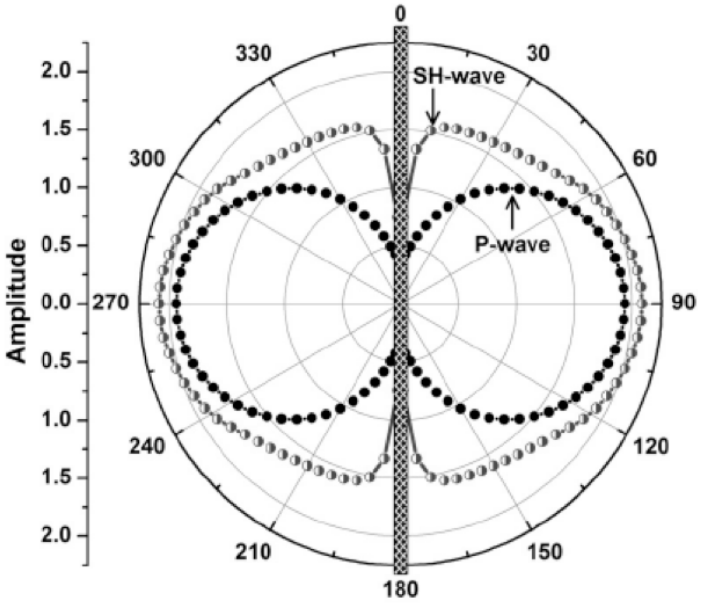

(c)

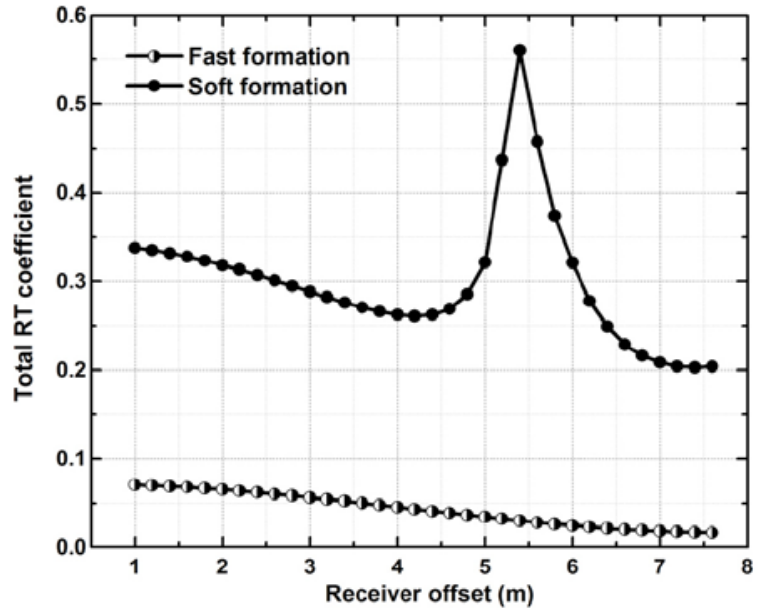

(d)

Fig. (9). Reflected P-wave amplitude comparison of fast formation and slow formation, the borehole reception pattern, reflection and transmission coefficient. (a) Relationship between reflected P-wave amplitude and receiver offset for various reflector orientations in a slow formation, (b) the borehole reception patterns for P-wave and SH-wave incidences in a slow formation, (c) the borehole reception patterns for Pwave and SH-wave incidences in a fast formation, (d) Total reflection and transmission coefficient of P-wave acoustic energy that radiates away from the borehole and reflects back to the borehole from near-borehole reflector versus receiver offset. 


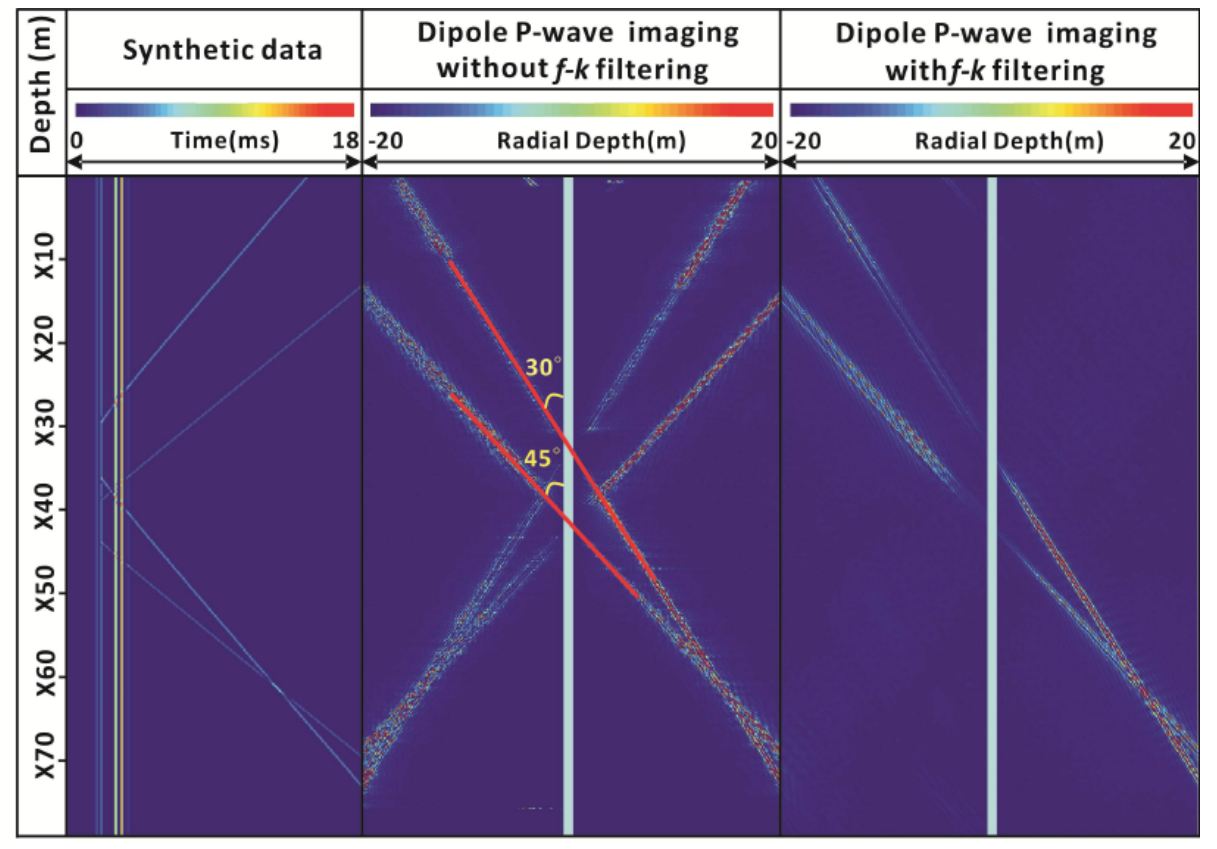

(a)

(b)

(c)

Fig. (10). Synthetic data example for $180^{\circ}$ uncertainty of reflector azimuth. (a) Variable-density display (VDL) of synthetic $x x$-component data calculated for receiver one of an eight-receiver array, (b) Imaging result obtained from raw acoustic data (Fig. 10a) without $f-k$ filtering, and (c) Imaging result with $f-k$ filtering.

minimum value, and subsequently their amplitude begin to rise slightly, and this variation becomes more gradual along with various source-reflector orientations from $\varphi=90^{\circ}$ to $\varphi=0^{\circ}$. The reflected $\mathrm{P}$-wave amplitude in the slow formation is about 3-4 times stronger than that in the fast formation within the scope of the existing dipole logging tool receiver offset. Reflected P-wave amplitude shows a monotonic increase for fixed receiver offset.

Fig. (9b and 9c) show the borehole reception patterns for $\mathrm{P}$-wave and $\mathrm{SH}$-wave incidences in the slow and fast formation, respectively. Our calculations are performed with a single frequency of $3 \mathrm{kHz}$ and an incident angle of $0^{\circ}$. Physical properties of medium used in the calculation are given in Table 1 (fast formation). For the slow formation (Fig. 9b), the wave field plane of $\mathrm{P}$-wave or $\mathrm{SH}$-wave incidence inside the borehole is much similar. Both reception patterns allow $\mathrm{P}$ - and $\mathrm{SH}$-wave to illuminate near-borehole reflectors at about $40^{\circ}-90^{\circ}$ dip angles. In contrast, for the fast formation $\mathrm{SH}$-wave coverage is better than that of $\mathrm{P}$-wave and approaches a unit circle. So we can use $\mathrm{SH}$-wave for reflection imaging in a fast formation. However, for a slow formation, both waves can be used for reflection imaging.

At last, we calculated the reflection and transmission coefficients [11] of the entire reflection system (radiation, reflection and reception). Fig. (9d) gives the transmission coefficient inside a borehole versus receiver offset, as we can see the coefficient in the slow formation is about 3-5 times stronger than that of the fast formation within the scope of the existing dipole logging tool receiver offset. The reason may be that the impedance contrast between the slow formation and the borehole fluid is much smaller compared to that of the fast formation, allowing for a larger portion of the acoustic energy to radiate into the formation.
It needs to be stressed that the application of P-wave remote imaging is limited to slow formation conditions where the dipole generated P-wave energy is prominent. Even so, it also can be applied to important scenarios. For example, it has the potential to obtain the geological structure information ahead of the drill bit during the drilling operation in the shallow sea; or it can be used to map geological boundaries intersecting a borehole. It can be an important supplement to improve the entire remote acoustic imaging system.

\section{$180^{\circ}$ Uncertainty of Reflector Azimuth}

It is worth noting that a $180^{\circ}$ uncertainty in the determination of reflector azimuth using dipole waves is inherent for the dipole source and receiver system. By replacing $\varphi$ with $\varphi+180^{\circ}$ into equations 3 or 4 , these equations results will stay the same. And thus the two angles $\left(\varphi\right.$ and $\left.\varphi+180^{\circ}\right)$ cannot be distinguished. For a near-borehole reflector, this means that we cannot determine whether the reflector is on the right $(\varphi)$ or left $\left(\varphi+180^{\circ}\right)$ side of the borehole (i.e., its dipping direction).

Fig. (10a) shows a variable-density display (VDL) of synthetic modeling $x x$-component data calculated for receiver one of an eight-receiver array. In the calculation, the source-receiver distance is $3.2 \mathrm{~m}$ and formation P- and Swave velocity are $2300 \mathrm{~m} / \mathrm{s}$ and $1250 \mathrm{~m} / \mathrm{s}$ (as shown in Table 1), respectively. For the convenience of discussing $180^{\circ}$ uncertainty, we use two bed reflectors, spaced $8.47 \mathrm{~m}$ apart, cross the borehole at $30^{\circ}$ and $45^{\circ}$, respectively. Reflected dipole $\mathrm{P}$-wave is extracted from the $x x$-component data, and an image of near-borehole reflectors is obtained by migration. Fig. (10b and 10c) show two different imaging results obtained from raw acoustic data (Fig. 10a) without (Fig. 10b) and with (Fig. 10c) $f$ - $k$ filtering [1], respectively. 
Obviously, there are two solutions satisfying equations 3 and 4 in the $0-180^{\circ}$ azimuth around the borehole. For Fig. (10b), bed boundary leaning to the left has signature just $180^{\circ}$ from the bed boundary leaning to the right, thus the two angles (i.e., dipping direction) cannot be distinguished in the process of dip stacking and migration. In the premise of known dip direction, we use $f-k$ filtering for the imaging result (bed boundary leaning to the right) of Fig. (10b), the result is shown in Fig. (10c). It can be seen that the imaging result agrees with our forward model very well.

In field applications, this azimuth ambiguity can be eliminated through other information such as a dip logging, electric or sonic image logging, analysis results of the geology, seismic profiles or cores outcrop description in connection with the borehole trajectory. Nevertheless, in many geological applications, such as fracture evaluation, knowing the strike of a geological reflector can provide important information (e.g., improvement the efficiency of hydraulic fracturing treatment in reservoirs by oriented fracturing).

\section{CONCLUSION}

An important advance recently in single-well reflection imaging is the use of a dipole acoustic system in a borehole to radiate and receive elastic waves to and from a remote geologic reflector in formation. A borehole dipole source can radiate a P-wave and two kinds of shear wave (i.e., SV- and $\mathrm{SH}$-wave) into the formation.

The theoretical analysis and numerical simulation demonstrate the radiation, reflection and reception characteristics of the wavefield generated by a borehole dipole source. In an acoustic slow formation the low-frequency wave data, as compared to conventional monopole data, greatly enhances the radial penetration depth of the image. P-wave generated by a dipole acoustic tool has high amplitude, obvious sensitivity and wide coverage attribute. It can also be used for remote reflection imaging, providing the theoretical basis for directional P-wave remote acoustic imaging. The P-wave (or $\mathrm{S}$-wave) from a cross-dipole logging data set can successfully yield the space position and strike azimuth of a nearborehole reflector, but not its dipping direction. In practice, dipping direction can be eliminated with other information. The comparison of the dipole $\mathrm{P}$-wave imaging and the conventional monopole $\mathrm{P}$-wave imaging demonstrates that dipole P-wave imaging has great potentials. Dipole P-wave imaging can fully meet requirements and serve as an important supplement to the remote acoustic imaging logging.

\section{ACKNOWLEDGEMENTS}

This work is supported by the National Science Foundation of China (No. 41404091 and NO. 41404100), Shandong Province Natural Science Foundation (ZR2014DQ004), COSL-UPC Allied Borehole Acoustic Laboratory, and New Well Logging Technology Laboratory for Complex Reservoir, China University of Petroleum.

\section{REFERENCES}

[1] B. E. Hornby, "Imaging of near-borehole structure using fullwaveform sonic data", Geophysics, vol. 54, no. 6, pp.747-757, 1989.

[2] H. Yamamoto, S. Watanabe, and H. Mikada, "Fracture imaging using borehole acoustic reflection survey", Proceedings of the $4^{\text {th }}$ SEGJ International Symposium, Kyoto, Japan, 1998, pp. 375-382.

[3] L. Chabot, D. C. Henley, and R. J. Brown, "Single-well imaging using the full waveform of an acoustic sonic", $71^{\text {st }}$ SEG Annual Meeting, Expanded Abstracts, San Antonio, 2001, pp. 420-423.

[4] X. M. Tang, H. Glassman, and D. Patterson, "Single-well acoustic imaging in anisotropic formations", Geophysics, vol. 73, no. 4, pp. D11-D16, 2008.

[5] D. Patterson, X. M. Tang, and J. Ratigan, "High-resolution borehole acoustic imaging through a salt dome", $78^{\text {th }}$ SEG Annual Meeting, Expanded Abstracts, Las Vegas, 2008, pp. 319-323.

[6] C. Esmersoy, C. Chang, and M. R. Kane, "Sonic imaging: a tool for high resolution reservoir description", $67^{\text {th }}$ SEG Annual Meeting, Expanded Abstracts, Dallas, 1997, pp. 250.

[7] X.M. Tang, Y. Zheng and D. Patterson, "Processing array acousticlogging data to image near-borehole geologic structures", Geophysics, vol. 72, no. 2, pp. E87-E97, 2007.

[8] Z.T. Wei, and X.M. Tang, "Numerical simulation of radiation, reflection, and reception of elastic waves from a borehole dipole source", Geophysics, vol. 77, no, 6, pp. 1-9, 2012.

[9] X.M. Tang, "Imaging near borehole structure using directional acoustic wave measurement", Geophysics, vol. 69, pp. 1378-1386, 2004.

[10] X.M. Tang, and D. Patterson, "Single-well S-wave imaging using multi-component dipole acoustic log data", Geophysics, vol. 74, no. 6, pp. 211-223, 2009.

[11] K. Aki, and P. Richards, Quantitative Seismology: Theory and Methods, W. H. Freeman \& Co., UK 1980.

[12] C. Peng, "Borehole Effects on Downhole Seismic Measurements", Ph.D. thesis, Massachusetts Institute of Technology, US, 1994.

[13] A. Ben-Menahem, and S. Kostek, "The equivalent force system of a monopole source in a fluid-filled open borehole", Geophysics, vol. 56, no. 9, pp. 1477-1481, 1991.

[14] K.R. Kelly, R. Ward, W.S. Treitel, andr.M. Alford, "Synthetic seismograms, a finite difference approach", Geophysics, vol. 41, pp. 2-27, 1976.

[15] X.M. Tang, and C.H. Cheng, "Quantitative Borehole Acoustic Methods", Elsevier, Amsterdam, 2004.

[16] Z.T. Wei, "Research on single-well acoustic imaging logging by using numerical modeling and experimental measurement", China University of Petroleum, Qingdao (East China), 2011.

\section{CONFLICT OF INTEREST}

The authors confirm that this article content has no conflict of interest.

\footnotetext{
Received: November 03, 2014

Revised: December 23, 2014

Accepted: June 23, 2015

(C) Wei et al.; Licensee Bentham Open.

This is an open access article licensed under the terms of the Creative Commons Attribution Non-Commercial License (http://creativecommons.org/licenses/by-nc/3.0/) which permits unrestricted, non-commercial use, distribution and reproduction in any medium, provided the work is properly cited.
} 